\title{
Avaliação da multifatorialidade para dependência química entre infantes e adolescentes no estado do Rio de Janeiro
}

\author{
Evaluation of the multifatorial influence for chemical \\ dependence between infants and teenagers in the state of Rio \\ de Janeiro, Brasil
}

\author{
Nicolau Maués Serra Freire'; Antelina Serra Freire Lôbo²; Stela Telles Oliveira ${ }^{3}$ \\ Resumo
}

Em 2005/06, um inquérito epidemiológico sobre a multifatorialidade para dependência química de crianças e adolescentes foi desenvolvido com 3.000 dependentes, em 28 municípios/8 regiões geográficas do Rio de Janeiro. Em cada município, Agentes Comunitários de Saúde e Mães Sociais selecionaram as amostras, com os requisitos: terem entre 4-16 anos, concordarem em ser voluntários, com a permissão dos responsáveis; terem paradeiro conhecido a mais de três anos no local. O questionário pré-elaborado foi respondido por todos, em forma livre de expressão. As opções de respostas de cada item foram transformadas em valores relativos percentuais, que foram comparados entre si pelo teste " $z$ ", arbitrandose alfa em $10 \%$. Os 12 descritores de maior moda foram: desagregação familiar quando o pai abandona o lar (20\%); desestruturação ou dissolução do núcleo familiar (14\%); dependência química nas famílias (13\%); prostituição materna (12\%); violência doméstica (7\%); maus tratos por parte do pai, da mãe, ou dos dois (6\%); pai ou mãe presidiário (6\%); falta de comprometimento dos pais biológicos $(5 \%)$; negligência dos responsáveis (5\%); abandono do lar por um ou dois genitores (4\%); abuso sexual do menor por pai, padrasto/madrasta, tio/tia, primo/prima etc. (4\%); mães solteiras que necessitam trabalhar (4\%). Concluiu-se que: a desestruturação familiar é o principal fator condicionante para que crianças e adolescentes se tornem usuários dependentes químicos; tanto o pai, quanto a mãe são fundamentais na formação da criança/adolescente com conhecimento para não se tornar dependente químico; a realidade do flagelo do uso de drogas no Rio de Janeiro tem identidade com as situações em outros países; a falta, e o excesso de recursos financeiros dos provedores das crianças e adolescentes, condicionam a falta de atenção para estes, favorecendo a entrada no mundo das drogas; as cinco razões básicas para atração dos jovens ou consumo de drogas divulgadas pela OMS, acontecem no Rio de Janeiro.

Palavras-chave: Dependência química. Criança e adolescente. Fatores condicionantes. Descritores de importância na causalidade. Rio de Janeiro.

\begin{abstract}
In 2005/06, an epidemiological study on multifactorial chemical dependency in children and adolescents was conducted with 3,000 addicts, in 28 cities/8 geographic regions of Rio de Janeiro. In each county, Community Health Workers and Foster Parents selected subjects with the following requirements: they had to be between 4-16 years old, agreed to be volunteers with the permission of those responsible; and have lived on location for three years or more. The prepared questionnaire was answered by everyone in free expression. The response options for each item were transformed into percentage figures, which were compared amongst them by test "z", determining alpha at $10 \%$. The 12 highest trend descriptors were: family breakdown when the father abandons the home (20\%); disruption or
\end{abstract}

\footnotetext{
${ }^{1}$ Bacharel em Medicina Veterinária; Especialização em Parasitologia Veterinária; Mestrado em Medicina Veterinária; Doutorado em Medicina Veterinária (UFRRJ). Médico Veterinário Pesquisador do Instituto Oswaldo Cruz (FIOCRUZ). E-mail: nmsf@ioc. fiocruz.br

${ }^{2}$ Médica no PSF do Município de Volta Redonda, RJ. E-mail: arldlobo@yahoo.com.br

${ }^{3}$ Bióloga da Secretaria de Saúde de Volta Redonda, RJ.
} 
dissolution of the family (14\%); chemical dependence in families (13\%); maternal prostitution (12\%); domestic violence (7\%); abuse by the father, mother, or both (6\%); father or mother imprisonment $(6 \%)$; lack of commitment from parents (5\%); parental neglect (5\%); abandonment by one or both parents (4\%); sexual abuse of minor by parent, stepparent, uncle/aunt, cousins etc. (4\%); and single mothers who need to work (4\%). It was concluded that the disruption of the family structure is the major risk factor for children and adolescents to become addicts. Both father and mother are fundamental in raising a child/adolescent who is able to avoid becoming a drug addict. The reality of drug use affliction in Rio de Janeiro relates with situations in other countries: the lack, and/or excess of financial resources of children and adolescents' providers lead to the lack of attention towards them, favoring entry into the world of drugs. The five main reasons youngsters are attracted into drug use, as released by WHO, take place in Rio de Janeiro.

Key-Words: Chemical dependency. Child and adolescent. Conditioning factors. Describers of importance in causality. Rio de Janeiro.

\section{Introdução}

A implantação do Sistema Único de Saúde $=$ SUS pela constituição brasileira firmou mais e melhor a responsabilidade do estado sobre a saúde da população, ao afirmar que saúde é direito de todos e dever do Estado (CORDEIRO, 1991; COSEMS, 1997). Na esteira desta política, buscouse consolidar as ações preventivas em tentativa clara de romper com o ciclo econômico das doenças. A filosofia de prevenção às doenças foi acompanhada pela proteção e promoção à saúde. Neste campo, programas foram idealizados, do qual se destacam o Programa Saúde na Família = PSF, uma evolução do Programa do Médico de Família, que necessitou de um apoio forte e ativo vindo da vigilância em saúde, com higiene e saúde pública, com marcante participação multiprofissional e característica interdisciplinaridade (CZERESNIA, 1997).

Muitas ações foram programadas, algumas implantadas, mas a visão holística da saúde promovida, protegida e fomentada, nem sempre está bem avaliada nas diferentes esferas sociais que integram a sociedade. Percebem-se algumas investidas isoladas de municípios, mas há carências de estudos pareados entre atividades municipais na unidade da federação, o estado. Muitas vezes, a raiz do problema é ainda mais primária, vem das chamadas famílias pré-aditivas, que possuem estrutura fragilizada geradora de patologias, e não conseguem perceber que sua dinâmica é aditiva e pode facilitar o desenvolvimento da drogadicção bem como de outras adicções (BLEFARI, 2002).

A Organização Mundial da Saúde apresentou cinco razões básicas pelas quais os jovens, de qualquer civilização contemporânea, podem ser atraídos para o consumo de drogas: a necessidade de se sentir adulto para tomar suas próprias decisões; a ânsia de ser "popular" entre seu grupo direto de convívio; a procura do relaxamento e bem estar de tranqüilidade; o desejo de conseguir vencer o medo, para ter coragem de correr riscos e rebelar-se; a simples curiosidade. Assim, tomam a decisão de experimentar a droga e, feita a escolha, crêem estar pensando que estão se responsabilizando por ela, e se acreditam mais livres.

Aqueles que decidem consumir uma droga estão fazendo uma opção, uma escolha. É claro que muitos fatores contribuem para que essa escolha se desse, com ou sem a consciência do próprio, mas a escolha, no final, foi da pessoa. Continuar usando drogas também é uma opção, mas cada vez menos controlável. Isso porque o organismo adapta-se à presença da droga, vai havendo modificações no cérebro. Quando o indivíduo fica sem a droga, passa a sentir-se muito mal, desconfortável, irritado, deprimido, ansioso. $\mathrm{O}$ dependente acha que o único alívio possível é a continuidade do consumo. Conforme a dependência se instala, a pessoa passa a 
abrir mão de coisas que antes eram muito importantes para si. É o momento em que aparecem as brigas e discussões com a família, a piora no desempenho escolar, a venda de objetos para comprar drogas. Tudo passa a girar em torno do consumo de drogas. A partir desse ponto, o indivíduo não consegue mais ficar sem usar drogas, e tem-se uma condição patológica (uma doença) que tira a liberdade de opção do indivíduo.

O flagelo do uso de drogas não está restrito ao Brasil, é um problema internacional de saúde como pode ser comprovado por pesquisas realizadas na: Oceania $=$ Austrália; Ásia $=$ Índia e Tailândia; na Europa $=$ Espanha; e América do Norte $=$ Estados Unidos, que apontam o uso de substâncias psicoativas como fator indutor da delinqüência (DURANT; KNIGHT; GOODMAN, 1997; CROWLEY et al., 1998; AMITI-MACKESY; FENDRICH, 1999; KUO et al., 2002; GONZALVO, 2002; BELENKO; LOGAN, 2003; HELSTROM et al., 2004; SWAHN; DONOVAN, 2004; KIM; KIM, 2005; LENNINGS; KENNY; NELSON, 2006).

Verificou-se a necessidade de vivenciar mais intensamente a causalidade do flagelo da dependência química que se alastrou pela sociedade brasileira, e em particular a carioca e fluminense. Houve a oportunidade de reunir um especialista em planejamento e análise de pesquisas biológicas, e interagir com um Programa de Saúde na Família, PSF, e com a estrutura administrativa. Com isso, houve a possibilidade de desenvolver um projeto de investigação com busca ativa, com a participação direta dos atores do agravo social, na tentativa de identificar, dentre os muitos fatores concorrentes na causalidade da dependência química, os 12 maiores indutores de crianças e adolescentes para consumo de drogas e a dependência química no estado do Rio de Janeiro.

\section{Material e métodos}

Foram reunidas as competências médica (adquirida por anos seguidos de trabalho no
Programa de Saúde na Família, em mais de um município brasileiro), biológica, administrativa (em atividades em saúde com comunidades e na Secretaria Municipal de Saúde), e epizootiológica (com planejamento e execução de pesquisa a campo, com análise estatística e interpretação dos resultados). A partir dessas competências, foi estruturado o trabalho e elaborado o plano de ação para o inquérito da endemia "uso de drogas".

Considerando a totalidade de regiões geográficas do estado do Rio de Janeiro e os espaços geopolíticos dos municípios que as compõem, o processo de amostragem foi por sorteio simples de 30 municípios para comporem a amostra de um trabalho individualizado (com crianças e adolescentes envolvidos por dependência química, observacional - não buscando imediatamente a modificação do "status quo"), transversal (com desenvolvimento em dois anos, 2005 e 2006). Isso permite caracterizar o trabalho efetuado como inquérito epidemiológico.

Foi definido um questionário com perguntas que permitissem a obervação de causas pela análise das respostas, de tal forma que o questionado responderia à mesma indagação feita de forma diferente, em momentos não seqüenciais, para observar a veracidade do dado e a impactação no agravo.

Em cada município, eram estabelecidos contatos com Agentes Comunitários de Saúde - ACS, ou com "Mães Sociais" de crianças e adolescentes dependentes químicos para entrevistar 100 destes jovens, utilizando a ficha questionário pré-estabelecida e igual para todos os 3.000 entrevistados (GOULART, 1996). Os contatados que integrariam a amostra para estudo tinham que corresponder a requisitos como: serem maiores de quatro anos, e menores de dezesseis; concordarem em ser voluntários, após a explicação do que era o programa, e autorizarem o uso dos dados para a análise e divulgação científica dos resultados, com o conhecimento e a permissão dos responsáveis; terem paradeiro conhecido a mais de três anos no 
local.

Os questionários eram preenchidos sempre pelas mesmas pessoas, que escutavam as respostas dadas pelo entrevistado, e faziam o registro no espaço próprio destinado no questionário para a resposta, assim evitando-se o viés de aferição ou de confundimento se fossem registrados por entrevistadores diferentes. Os entrevistados e seus responsáveis eram informados do que se tratava o estudo, e quando aceitavam voluntariamente participar sem qualquer forma de remuneração, e davam permissão para uso das respostas na análise situacional, eram incluídos no programa da pesquisa.

Os questionamentos com as perguntas já formuladas eram realizados em forma de conversa livre, descontraindo o entrevistado sem indução de resposta. Assim, do universo de respostas possíveis de serem dadas, os entrevistadores deveriam enquadrá-las de acordo com as alternativas dos indicadores de saúde pré-estabelecidos para os quesitos inquiridos.

Foi fixado o número de entrevistados com base no Projeto Piloto, quando se avaliou a variabilidade dos resultados e arbitrou o erro aceitável do tipo $1(\alpha=10 \%)$, com estimativa de que as entrevistas se completassem em dois anos (2005-2006). Os formulários brutos foram triados, e quando foi encontrada qualquer não conformidade, a entrevista era descartada e buscava-se um novo jovem para ser entrevistado, em substituição ao questionário descartado (HOCHMAN, 1998).

Dos formulários aprovados na triagem foram tabuladas as opções de respostas de cada item e transformadas em valores relativos percentuais, que foram comparados entre si por teste analítico. Os itens foram analisados estatisticamente pelo teste " $\mathrm{z}$ " para comparação entre duas proporções, com base no mesmo nível de significância arbitrando $(10 \%)$ para a tomada de decisão (SERRA-FREIRE, 2002). Os resultados foram classificados ordinalmente pelo valor modal das percentagens, e são apresentados os 12 indicadores de maior valor impacto na composição multifatorial da dependência química, conforme a proposta inicial do trabalho.

\section{Resultados e discussão}

Dos 30 municípios selecionados, em dois não foi possível conseguir voluntários para participar do estudo, por diferentes razões que aqui não cabe comentar. É fato, porém, que existiam crianças e adolescentes envolvidos com dependência química.

Para manter os 3.000 questionários programados para o estudo, para os 28 municípios que foram trabalhados, ampliou-se para 107 o número dos entrevistados, e em um identificado por sorteio simples, escolheu-se Niterói para nele serem entrevistados 111 jovens.

Do total de indicadores obtidos, os doze mais freqüentes variaram entre $20 \%$ e $4 \%$, e os descritores estão apresentados no Quadro 1. Comparados estatisticamente, o primeiro descritor diferiu significativamente dos outros onze; o segundo, terceiro, e quarto não diferiram entre si, mas diferiram dos demais. Do quinto ao nono descritor, a diferença, quando ocorreu, não foi significativa, mas o quinto, sexto e sétimo diferiram significativamente do décimo, décimo primeiro e décimo segundo, e estes não diferiram entre si, nem com o oitavo e o nono descritor.

A análise social destes indicadores revela que até seria válido reunir os quatro primeiros itens dentro de tema "degradação social familiar" incluindo o abandono paterno aos filhos e o desvinculamento arbitário do dever de orientar, cuidar, educar e suprir as necessidades do descendente. Isso ocorria porque o núcleo convivente da família, representado por avós paterno e materno, tios e primos não mantém relações sociais de equilíbrio emocional, e sentimental, ou alternam momentos de carinho e compreensão com outros de repúdio e agressão, motivados por dependência de alcoolismo ou de outras drogas, problemas de inserção social na 
Quadro 1 - Relação percentual dos 12 principais descritores de fatores condicionantes para que crianças e adolescentes passem a dependentes químicos no estado do Rio de Janeiro, em amostra de 3.000 participantes de 28 municípios, trabalhada em 2005-2006.

\begin{tabular}{|c|c|c|}
\hline $\begin{array}{l}\text { Número de } \\
\text { ordem }\end{array}$ & Fator condicionante à dependência química(descritor) & $\begin{array}{l}\text { Percentagem } \\
(\%)\end{array}$ \\
\hline 01 & $\begin{array}{l}\text { Desagregação familiar em que o pai abandona o lar e estabelece } \\
\text { uma } 2^{\mathrm{a}} \text { e até a } 3^{\mathrm{a}} \text { união. }\end{array}$ & $20^{\mathrm{a}}$ \\
\hline 02 & $\begin{array}{l}\text { Desestruturação ou dissolução do núcleo familiar, pais, avós, tios } \\
\text { e primos que convivem no mesmo ambiente sem se relacionar } \\
\text { socialmente. }\end{array}$ & $14^{\mathrm{b}}$ \\
\hline 03 & $\begin{array}{l}\text { Dependência química das famílias, sendo mais freqüente o } \\
\text { alcoolismo (responsável que passa o dia inteiro alcoolizado, } \\
\text { deixando a responsabilidade dos afazeres domésticos para o } \\
\text { adolescente ou a criança). }\end{array}$ & $13^{\mathrm{b}}$ \\
\hline 04 & Mães que se prostituem. & $12^{\mathrm{b}}$ \\
\hline 05 & $\begin{array}{l}\text { Violência doméstica física e psicológica (brigas diárias e } \\
\text { contínuas entre os pais ou responsáveis) }\end{array}$ & $7^{\mathrm{c}}$ \\
\hline 06 & Maus tratos por parte dos pais ou responsáveis. & $6^{c}$ \\
\hline 07 & Pais presidiários & $6^{c}$ \\
\hline 08 & $\begin{array}{l}\text { Falta de comprometimento dos pais biológicos com seus filhos } \\
\text { (não cuidam nem nos fins de semana, quando não trabalham, } \\
\text { deixando-os nas ruas, sem higiene e sem controle). }\end{array}$ & $5^{\mathrm{cd}}$ \\
\hline 09 & Negligência ou omissão do pai/mãe ou padrasto/madrasta & $5^{\mathrm{cd}}$ \\
\hline 10 & Abandono do lar por um ou pelos dois genitores & $4^{\mathrm{d}}$ \\
\hline 11 & $\begin{array}{l}\text { Abuso sexual do menor por pai, padrasto/madrasta, tio/tia, } \\
\text { primo/prima etc. }\end{array}$ & $4^{\mathrm{d}}$ \\
\hline 12 & $\begin{array}{l}\text { Mães solteiras que necessitam trabalhar para o sustento dos filhos } \\
\text { e não tem com quem deixá-los, ficando estes pelas ruas, mas se } \\
\text { preocupam com eles e os cuidam quando não trabalham. }\end{array}$ & $4^{\mathrm{d}}$ \\
\hline
\end{tabular}

Obs: Na coluna percentagem, expoentes de letras diferentes indicam diferença estatística significativa $(\alpha=10 \%)$; letras iguais indicam diferença não significativa.

comunidade, ou mesmo por prostituição sexual. Esses fatores corresponde a $59 \%$ dos fatores condicionantes para que crianças e jovens sejam iniciados e permaneçam na dependência química. Essa verificação nem sempre é fácil, porque a dependência química é a doença da negação: embora esteja cheio de problemas nas principais áreas de sua vida, o dependente nega que tem um problema, ou atribui a culpa a outros, e não aceita ajuda. E sabemos que, quando mais culpamos os outros por nossas escolhas erradas, menos autônomos e mais dependentes ficamos. A degradação social familiar é causa de grande sofrimento, e, de acordo com Freitas (2002), o adolescente é extremamente vulnerável aos apelos provenientes do mundo das drogas em virtude das modificações pelas quais passa o seu mundo interno. A fase da adolescência é muito complexa, com ganhos e perdas importantes. A negação desse sofrimento traduz-se em uma das graves patologias desse período da vida do ser humano. Essa negação, muitas vezes, conduz a comportamentos anti-sociais e autodestrutivos, encobridores de uma intensa angústia existencial.

A repetência nas atividades escolares e a evasão 
escolar, apontadas como possíveis causas, não chegaram nem a dois por cento das respostas, sinalizando que os próprios comprometidos com a dependência entendem que estas são conseqüências, e não causas para o agravo.

No segmento da população completamente excluído, não integrante de qualquer esfera social, os descritores 8, 9 e 10 (Quadro 1) estão mais presentes. Esse segmento e as esferas da comunidade de base, e da pastoral têm maior participação cultural no ciclo econômico da doença, e atribuem grande parcela de culpa aos problemas de saúde à falta de hospitais, por desconhecerem os princípios do SUS e do PSF.

$\mathrm{Na}$ esfera social de partidarismo os descritores que predominaram nas respostas das crianças e adolescentes dependentes químicos foram os relacionados à violência (itens 5, 6 e 11 no Quadro 1). São encarados como ações violentas tanto as agressões físicas com força desmedida, em qualquer parte do corpo (principalmente o rosto e o crânio), a pressão psicológica constante com ameaças de castigos com humilhação ou com cárcere, o desrespeito contínuo entre os familiares demonstrados através de palavras e gestos, e os abusos sexuais no intra e peridomicílio, tanto masculino como feminino.

Ações das normas e programas governamentais poderiam minimizar estes fatores se revissem os procedimentos para o funcionamento privilegiando o ciclo econômico e dinâmico da saúde (Fig. 1). A sociedade, os serviços de pesquisa e extensão das Instituições de Ensino Superior poderiam contribuir muito se efetuassem maior número de pesquisas e difundissem com maior presteza e assiduidade os resultados obtidos neste tipo de estudo, como o da pesquisa realizada no município de Porto Alegre (FERIGOLO et al., 2004), que mostrou dados da nossa realidade. Quanto mais precoce é a iniciação no consumo do álcool e do tabaco, maior é a vulnerabilidade para o uso de drogas ilícitas, e a probabilidade de marginalização por violência, e esses dados que corroboram com os dados encontrados na literatura internacional. É necessária melhor fiscalização das operações idealizadas e projetadas para a vigilância em saúde, e rever as programações ou reposicionar os programas em atividades, e liberar mais recursos humanos e materiais para que esses programas alcancem à finalidade para a qual foram aprovados. É preciso, ademais ampliar a liberação de bolsas auxílio de forma paternalista, descomprometida com o acompanhamento sério eficiente. Observa-se que existiram tentativas de ordenação, com vários programas criados como: $\mathrm{PSF}=$ Programa Saúde na Família, PNI = Programa Nacional de Imunização, PAI = Programa Ampliada de Imunização, PSM = Programa de Saúde da Mulher, PST = Programa de Saúde do Trabalhador, PSA = Programa de Saúde Animal, e outros atrelados ao Setor de Saúde que mantém interface com outros setores como o de Ecologia, Planejamento Urbano, e Setor Social, mas alguns tiveram grande eficácia, mas baixa eficiência. É o caso do Planejamento Urbano, em que há sérios problemas de transporte, segurança, lazer, saneamento que afetam diretamente a saúde e podem ser causa de desajustes sociais e, não são associados aos dados revelados por setores da investigação em saúde social, e isso desrespeita o SUS. Esse fato pode ser comprovado pelo levantamento realizado pelo Centro Brasileiro de Informações sobre Drogas (CARLINI et al., 2007), quando foi revelado ter havido, em cinco anos, um aumento de $30 \%$ na ingestão de bebidas alcoólicas entre jovens de 12 a 17 anos, e de $25 \%$ entre jovens de 18 a 24 anos.

O sétimo descritor (Quadro 1) aparece em situação conflituosa, enquanto uma parte dos jovens lamenta pela ausência do genitor, pai ou mãe presidiário, outra parte (um pouco menor) se alegra pela mesma razão. Na tentativa de entender os dois comportamentos díspares, chegou-se às seguintes explicações: os que lamentavam o fazem porque sentiam a ausência, a falta de cuidados, de carinho e de proteção, e pouquíssimos aludiram a problemas econômicos; os que se alegravam eram porque 


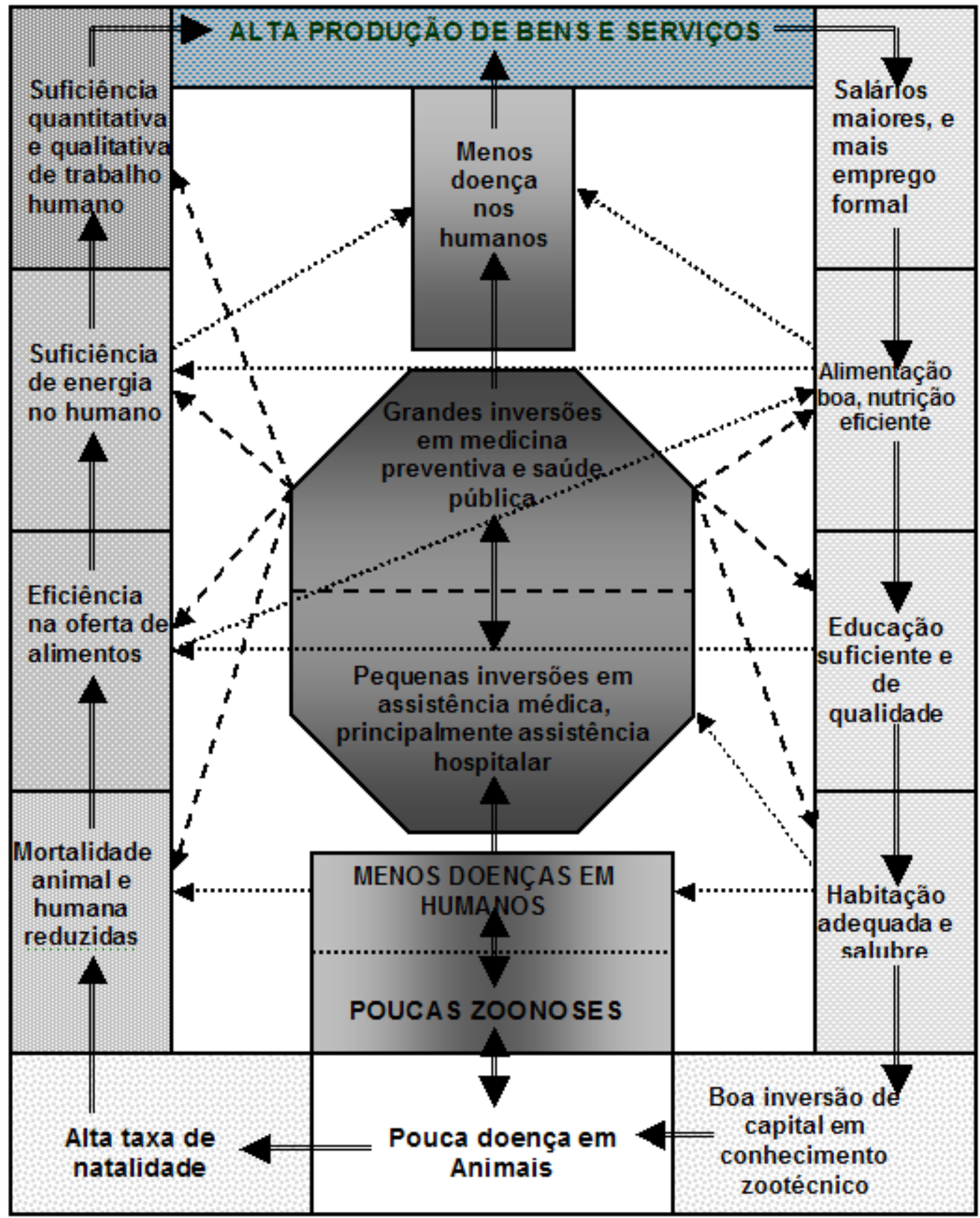

Figura 1. Ciclo Econômico e dinâmico da Saúde que precisa ser enraizado culturalmente na sociedade brasileira em geral, e na do estado do Rio de Janeiro em particular, fluxograma das interdependências.

estavam livres das constantes agressões, inclusive sexuais, mas a maioria destes ficava alegre porque havia maior disponibilidade de recursos financeiros.

O descritor 12 do Quadro 1 foi mais sensível nas esferas sociais mais organizadas, e inclui mais adolescentes com ensino de segundo grau incompleto, e até de formação profissional, envolvendo uma outra percepção de si próprio no contexto familiar e social. Chegaram à dependência 
química por desespero, em conseqüência da falta de apoio solicitado para familiares ou não. Nesse caso, há pessoas não comprometidas com o bem estar do solicitante que o induzem à fuga pela droga dos problemas de gravidez na adolescência.

É preciso valorizar o ciclo econômico da saúde (Fig. 1), enraizando culturalmente esta noção na sociedade, como uma nova ordem para a integração entre todas as esferas da sociedade, pois a dependência química é um agravo que se socializa com grande facilidade.

Neste ponto, cabe uma reflexão fundamental sobre estes dois descritores. No sétimo: por que havia maior quantidade de recursos financeiros? $\mathrm{O}$ genitor, genitora ou ambos, quando em liberdade, consumiam mais o numerário familiar? Havia menor despesa na família por conta da saída de um ou dois membros desta? Havia melhor gestão dos recursos disponíveis pela ausência do que estava preso? Ou havia aporte extra de recursos financeiros de origem por eles desconhecida, por conta do estado de presidiário? Essas possibilidades apareceram durante as conversas com os entrevistados, mas precisam de uma investigação diferenciada, que não é o objetivo deste trabalho. Do outro lado está o décimo segundo descritor, que mostra ser a suficiência de poder econômico um dos principais fatores condicionantes para esta causalidade. Cabe verificar como a criança e o adolescente encaram a sua relação social com os outros na base da relação mercantil, comprando álcool e vendendo, objetos, materiais e consciências. Mas quando o poder do dinheiro não pode resolver a situação, a criança e o adolescente se perdem, pois não têm estrutura moral e social. Uma questão levantada por todas as pesquisas da dependência química é o uso de álcool e drogas estar relacionado com a delinqüência em adolescentes, que evolui com problemas de conduta e traz a maior probabilidade de usar drogas (HEIM; ANDRADE, 2008). A estruturação dos núcleos familiares, em qualquer das esferas sociais, parece ser o melhor caminho para, a médio e longo prazo vencer o flagelo social da dependência química.
O item 1 do Quadro 1, descritor de desagregação familiar em que o pai abandona o lar e estabelece a segunda ou a terceira união, revelou ser o fator mais intenso na trama multifatorial da causalidade na dependência química. $\mathrm{O}$ impacto do abandono da criança até os dois anos de vida revelou menor porcentagem de indução à violência que o abandono de crianças com três ou quatro anos. Este, por sua vez, foi menor que o abandono de crianças com mais de quatro e menos de oito anos. Este resultado evidencia a importância do pai, ou da referência masculina na formação da personalidade ou do desenvolvimento social da criança, e sinaliza a necessidade da valorização desta figura para a família, e que ele próprio aprenda a valorizarse e assumir seu compromisso da educação pelo exemplo, reduzindo o risco da dependência química para os descendentes. Esse resultado tem identidade com as afirmações de Horta, Horta e Pinheiro (2006), que discutem as possibilidades da família proteger o adolescente do uso de drogas lícitas e ilícitas ou, de forma contrária, expô-los a elas.

Uma vez que ouso de álcoolélegal e aceito durante a vida adulta, os adolescentes estão constantemente expostos a normas sociais de aceitação do seu consumo. Assim, a clara expressão ou omissão por abandono, ou por negligência dos pais em relação ao consumo de álcool pelo adolescente, parece ser muito importante para estabelecer padrões de comportamento. Já para inibir o uso de maconha, parece ser importante que os pais desenvolvam recursos eficientes na monitoração da vida de seus filhos, o que, por si, pode encorajá-los a endossar as normas sociais já prevalentes que condenam o uso da maconha, menos comum tanto nas esferas sociais carentes, como nas muito abastadas de recursos financeiros (KOSTERMAN et al., 2000).

Verifica-se, então, que o desafio do combate ao uso de drogas, passa necessariamente pelo debate acerca das drogas no Brasil. Não basta discutir se devemos afrouxar as leis da maconha, mas também fazer um debate informado e com dados, e produzir uma política de drogas racional e balanceada, que 
possa ser avaliada constantemente (DIEHL REIS; LARANJEIRA, 2008). A implementação dessa política não ocorre espontaneamente, mas decorre uma ação determinada de governo. Talvez seja inútil esperarmos por uma grande política nacional de drogas, porém as ações locais de governo poderiam fazer uma grande diferença, pois os estados e municípios deveriam envolver-se nessas ações com a ajuda comunitária. A sociedade civil já está bastante mobilizada com o assunto álcool e drogas, porém é necessário que os governos democraticamente eleitos mostrem a sua capacidade de organizar uma resposta racional a um problema que afeta milhões de brasileiros com um custo enorme para o país.

\section{Conclusão}

Do inquérito epidemiológico acerca da dependência química no Estado do Rio de Janeiro, confrontado com dados de literatura, concluise: a desestruturação familiar é o principal fator condicionante para que crianças e adolescentes se tornem usuários dependentes químicos; tanto a figura masculina do pai, como a feminina da mãe são fundamentais na formação da criança/ adolescente capaz de saber discernir entre tornar ou não dependente químico; a realidade do flagelo do uso de drogas no Rio de Janeiro tem identidade com as situações em outros países; a falta, e o excesso de recursos financeiros dos provedores das crianças e adolescentes condicionam a falta de atenção para estes, e favorecem a entrada no mundo das drogas; as cinco razões básicas para atração dos jovens ou consumo de drogas divulgadas pela OMS, acontecem no Rio de Janeiro. Orientar a família na sua estruturação, e na educação pelo exemplo reduzirá dependência química entre seus descendentes.

\section{Referências}

AMITI-MACKESY, M. E.; FENDRICH, M. Inhalant use and delinquent behavior among adolescents: a comparison of inhalant users and other users. Addiction, Oxfordshire, v. 94, n. 4, p. 555-564, 1999.

BENSEÑOR, I. M.; LOTUFO, P. A. Epidemiologia: abordagem prática. São Paulo: Sarvier, 2005.

BLEFARI, A. L. A família e a drogadicção. 2002. Trabalho de conclusão de Curso (Aprimoramento em Dependência Química) - Universidade de São Paulo, Faculdade de Medicina, São Paulo.

CARLINI, E. A.; GALDURÓZ, J. C.; NOTO, A. R.; CARLINI, C. M.; OLIVEIRA, L. G.; NAPPO, A. S.; MOURA, Y. G.; SANCHEZ, Z. V. D. M. II levantamento domiciliar sobre o uso de drogas psicotrópicas no Brasil: estudo envolvendo as 108 maiores cidades do país - 2005. São Paulo: Páginas \& Letras, 2007.

CORDEIRO, H. Sistema único de saúde. Rio de Janeiro: Ayuri, 1991.

COSEMS, R. J. Manual do gestor SUS. Rio de Janeiro: Conselho dos Secretários Municipais de Saúde do Estado do Rio de Janeiro, 1997.

CROWLEY, T. J.; MIKULICH, S. K.; MACDONALD, M.; YOUNG, S. E.; ZERBE, G. O. Substance-dependent, conduct-disordered adolescent males: severity of diagnosis predicts 2-yar outcome. Drug and Alcohol Dependence, Limerick, v. 49, n. 3, p. 225-237, 1998.

CZERESNIA, D. Do cantágio à transmissão: ciência e cultura na gênese do conhecimento epidemiológico. Rio de Janeiro: Fiocruz, 1997.

DIEHL REIS, A.; LARANJEIRA, R. Halfway house for alcohol dependents: from theoretical bases to implications for the organization of facilities. Clinics, São Paulo, v. 63, n. 6, p. 827-832, 2008.

DURANT, R. H.; KNIGHT, J.; GOODMAN, E. Factors associated with aggressive and delinquent behaviors among patients attending an adolescent medicine clinic. Journal of Adolescent Health, New York, v. 21, n. 5, p. 303-308, 1997.

FERIGOLO, M.; BARBOSA, F. S.;ARBO, E.; MALYSZ, A. S.; STEIN, A. T.; BARROS, H. M. T. Drug prevalence at febem, Porto Alegre. Revista Brasileira de Psiquiatria, São Paulo, v. 26, n. 1, p. 9-15, 2004.

FREITAS, L. Adolescência, família e drogas: a função paterna e a questão de limites. Rio de Janeiro: Mauad, 2002. 
GONZALVO, G. O. Estado de salud de jóvenes varones delincuentes. Aten Primaria, Barcelona, v. 29, n. 7, p. 421-424, 2002.

GOULART, F. A. A. Municipalização: veredas, caminhos do movimento municipalista de saúde. Rio de Janeiro: Abrasco, 1996.

HEIM, J.; ANDRADE, A. G. Estudo do uso do álcool e das drogas ilícitas no comportamento de adolescentes de risco: uma revisão das publicações científicas entre 1997 e 2007. Revista de Psiquiatria Clínica, São Paulo, v. 35, supl. 1, p. 61-64, 2008.

HELSTROM, A.; BRYAN, A.; HUTCHISON, K. E.; RIGGS, P. D.; BLECHMAN, E. Tobacco and alcohol use as an explanation for the association between externalization behavior and illicit drug use among delinquent adolescents. Prevention Science, New York, v. 5, n. 4, p. 267-277, 2004.

HOCHMAN, G. A era do saneamento. São Paulo: Hucitec, 1998.

HORTA, R. L.; HORTA, B. L.; PINHEIRO, R. T. Drogas: famílias que protegem e que expõem adolescentes ao risco. Jornal Brasileiro de Psiquiatria, Rio de Janeiro, v. 55 , n. 4, p. 268-272, 2006.

KIM, H. S.; KIM, H. S. Gender differences in delinquent behavior among Korean adolescents. Child Psychiatry and Human Development, New York, v. 35, n. 4, p. 325$345,2005$.
KOSTERMAN, R.; HAWKINS, J. D.; GUO, J.; CATALANO, R. F.; ABBOTT, R. D. The dynamics of alcohol and marijuana initiation: patterns and predictors of first use in adolescence. Amererican Journal of Public Health, New York, v. 90, n. 3, p. 360-366, 2000.

KUO, P. H.; YANG, H. J.; SOONG, W. T.; CHEN, W. J. Substance use among adolescents in Taiwan: associated personality traits, incompetence, and behavioral/ emotional problems. Drug Alcohol Dependence, Lausanne, v. 67, n. 1, p. 27-39, 2002.

LENNINGS, C. J.; KENNY, D. T.; NELSON, P. Substance abuse and treatment seeking in young offenders on community orders. Journal of Substance Abuse Treatment, New York, v. 31, n. 4, p. 425-432, 2006.

SERRA-FREIRE, N. M. Planejamento e análise de pesquisas parasitológicas. Niterói: EdUFF, 2002.

SWAHN, M. H.; DONOVAN, J. E. Correlates and predictors of violent behavior among adolescent drinkers. Journal of Adolescent Health, New York, v. 34, n. 4, p. 480-492, 2004.

Recebido em 25 de junho de 2009 - Received on June 25, 2009 Aceito em 17 de maio de 2010 - Accepted on May 17, 2010. 